\title{
1 Viola: a structural variant signature extractor with user-defined classifications
}

2 Itsuki Sugita ${ }^{1,2}$, Shohei Matsuyama ${ }^{2}$, Hiroki Dobashi ${ }^{2}$, Daisuke Komura ${ }^{1, *}$ and Shumpei Ishikawa ${ }^{1, *}$

$3{ }^{1}$ Department of Preventive Medicine, Graduate School of Medicine, The University of Tokyo, 7-3-1 Hongo,

4 Bunkyo-ku, 1130033, Tokyo, Japan

$5 \quad{ }^{2}$ Faculty of Medicine, Tokyo Medical and Dental University, 1-5-45 Yushima, Bunkyo-ku, 1138510, Tokyo,

6 Japan

$7 \quad$ *Correspondence: kdais-prm@m.u-tokyo.ac.jp, ishum-prm@m.u-tokyo.ac.jp

\section{Summary}

9 Here, we present Viola, a Python package that provides structural variant (SV; large scale genome

10 DNA variations that can result in disease, e.g., cancer) signature analytical functions and utilities for 11 custom SV classification, merging multi-SV-caller output files, and SV annotation. We demonstrate 12 that Viola can extract biologically meaningful SV signatures from publicly available SV data for cancer 13 and we evaluate the computational time necessary for annotation of the data.

15 Availability: Viola is available on pip (https:/pypi.org/project/Viola-SV/) and on GitHub

16 (https://github.com/dermasugita/Viola-SV).

17 Contact: kdais-prm@m.u-tokyo.ac.jp

1. Introduction

20 Somatic mutations in cancer are the cumulative result of DNA aberrations caused by diverse mutational

21 processes. Recently, large scale studies of human cancer have revealed characteristic patterns of mutation

22 types, i.e., mutational signatures, arising from specific processes of single nucleotide variant formation. These

23 studies often provide theoretical explanations for known mutational processes and their consequences, e.g.,

$24 \mathrm{C}>\mathrm{A}$ substitutions and $\mathrm{CC}>\mathrm{TT}$ alterations caused by smoking and ultraviolet light exposure, respectively.

25 Structural variants (SVs) are another type of DNA mutation, defined as events larger than 50-bp in size or

26 involving multiple chromosomes, occupying non-negligible proportions of mutations in cancer cells (Mills et

27 al., 2011; Yi and Ju, 2018). Signature analysis of SVs may potentially provide novel insights into 
carcinogenesis. The development of high-throughput sequencing technologies and powerful SV callers has

29 improved the accuracy of SV event identification. Several mechanisms of SV formation have also been

30 identified (Yi and Ju, 2018). Therefore, research on SV signatures is gradually becoming realistic.

31 To date, several attempts have been made to decompose SV patterns into SV signatures, but an established

32 method has yet to be realized. Previous studies have mainly classified SVs according to segment size and

33 revealed an association between small tandem duplications and BRCA1 mutations (Li et al., 2020; Nik-Zainal

34 et al., 2016). However, a consensus has not been achieved on a precise SV classification method.

35 SVs can be classified by metrics other than length. Li et al. (2020) also used replication timing and common

36 fragile sites (CFSs). Interestingly, the biological meaningfulness of replication timing and CFSs has been

37 reported, e.g., the signatures of medium-sized (50-500 kb) tandem duplications occurring at the site of late

38 replication timing have been associated with CDK12 driver mutations, whereas CFS signatures have been

39 associated with gastrointestinal cancer. Other SV classification methods, such as microhomology and

40 association of transposons, have yet to be considered in detail; therefore, further analysis is required to

41 identify a suitable SV classification method for signature analysis.

42 At present, very few tools are available for SV signature analysis. To the best of our knowledge,

43 pyCancerSig (Thutkawkorapin et al., 2020), which is the first tool that can handle SVs for cancer mutation

44 signature analysis, is the only SV signature analysis tool currently available. However, pyCancerSig has

45 limitations in SV classifications as it only supports traditional SV classes, i.e., deletion, duplication, inversion,

46 and translocation, and length-based classification.

47 The time-consuming nature of parsing variant call format (VCF) files is also an obstacle to SV analysis.

48 VCF is the de facto standard format by which genetic variant data are recorded with high human readability.

49 However, from a data management perspective, VCF can be a bottleneck for analysis owing to its complex

50 structure. For SVs in particular, accurate interpretation of VCF records at the single nucleotide level requires

51 considerable learning costs. Difficulties with VCF interpretation cannot be ignored because even a 1-bp error

52 in positioning SVs can have critical consequences, e.g., in microhomology analysis.

53 Merging SV calls from different callers is also an issue in SV analysis. Precision of SV detection can be

54 improved by merging the results of multiple SV callers (Cameron et al, 2019; Kuzniar et al., 2020); however,

55 different SV callers use different ways to represent VCF files, which makes integration challenging. 
Here, we present Viola, a highly customizable and flexible Python package that supports SV signature

57 analysis with user-defined SV classification, matrix-generation functions, and a file exportation system that is

58 compatible with external statistical utilities and facilitates interpretation of results. Viola accepts VCF files

59 from four popular SV callers, namely Manta, Delly, Lumpy, and Gridss, and can also read BEDPE format

60 (Cameron. et al., 2017; Chen et al., 2016; Layer et al., 2014; Rausch et al., 2012). Viola also provides an

61 intuitive VCF file manager for filtering, annotating, converting VCF to BEDPE, and multicaller merging.

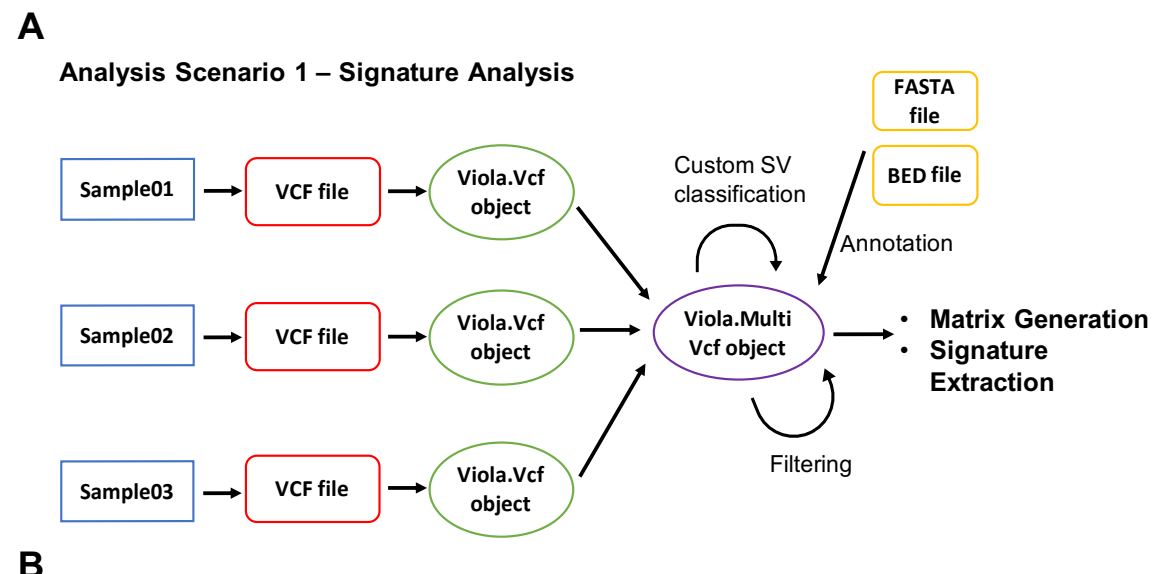

B

Analysis Scenario 2 - VCF merging

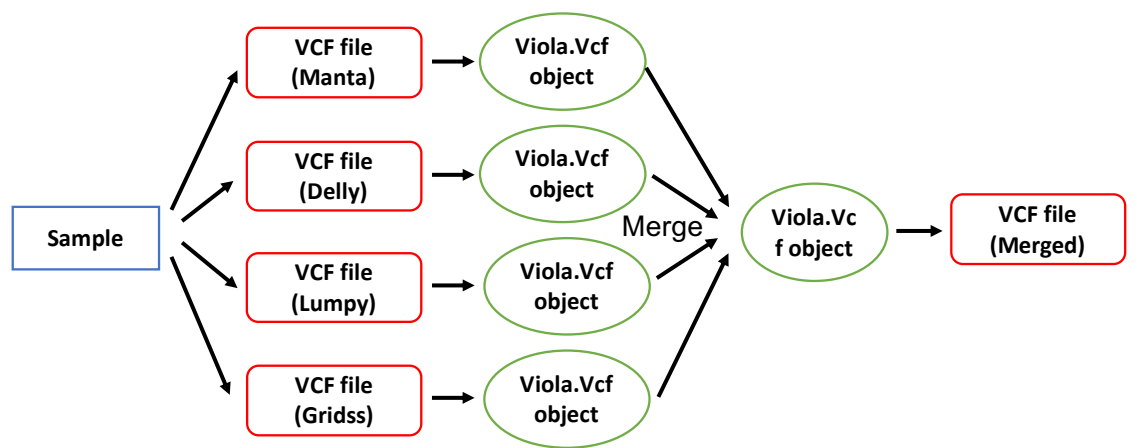

62 Fig.1. Visualization of the data flow in the main analysis scenarios. (A) Process of feature matrix

63 generation from multiple samples. (B) Overview of VCF merging system.

\section{Implementation}

\subsection{Data Structure}

67 Viola converts input SV data files, such as VCF and BEDPE files, into our original Python classes.

68 Instances of these classes store SV data as a set of tidy rectangular tables linked via identifiers such as SV ID

69 output by the SV callers (Supplementary Figure S1). These tables follow the principles of tidy data, i.e., each

70 SV record is a row, each variable is a column, and each type of observational unit is a table (Wickham, 2014). 
71 Consequently, storage of multiple values in one element is avoided, in contrast to the INFO and FORMAT

72 columns of a VCF file. Hence, a specific single value can be accessed by simply specifying the row and

73 column of the table of interest; this provides freedom in data handling without the need for cumbersome

74 codes.

Viola is written in the Python. Although it is intended for use within Python scripts, some features are available from the command line.

Viola supports SV signature analysis with user-defined SV classes (Figure 1A; Supplementary Figure S1A,

B). A simple feature matrix based on traditional SV types and SV length, output by the SV caller can be generated from the command line. Advanced uses such as annotation, filtering, and multicaller intersection, which are required to generate a complex feature matrix, are supported within Python scripts. In combination with these functions, it is possible to define a wide variety of SV classes, such as "Duplications located on

84 CFS sites" and "Deletions less than $50 \mathrm{~kb}$ in size, located on the early replication timing zones." These 85 operations can be implemented with simple syntax and are designed to refine the SV classification by trial and error (Supplementary Figure S2B).

87 From an internal data structure perspective, user-defined SV classes are interpreted as new INFO entries of

88 the VCF file. Hence, users can output new VCF or BEDPE files with annotation of novel SV classes as well

89 as generating a signature-analysis-ready feature matrix according to these additional SV classes.

90 Alongside signature analysis, Viola has the following features:

91 - Support of well-known SV callers including Manta, Delly, Lumpy, and Gridss. The notation has been 92 unified as much as possible to facilitate subsequent processing including merging (Figure 1B).

93 - Fast annotation methods that utilize the interval tree algorithm. Source files in BED format are 94 acceptable; thus, information such as gene names, CFSs, replication timing, and copy number can be 95 annotated if they can be expressed in BED format.

96 An intuitive method for filtering SV records. In addition to filtering for genomic coordinates and INFO 97 fields, filtering for FORMAT fields is possible. 
- Estimations of the length and sequence of microhomology from SV breakpoint positions. Where SV callers do not return microhomology information or publicly available SV data does not contain such information, Viola can estimate microhomology using the reference sequence.

101 The use of these characteristics is described in detail in the official Viola documentation, which is available online (https://dermasugita.github.io/ViolaDocs/docs/html/index.html).

\subsection{Custom SV Classification Overview}

105 With Viola, any information in the INFO field of the VCF can be used for SV classification. Many SV 106 callers write the SV type and length in the INFO field by default making it easy to classify by these variables. 107 For BEDPE files that do not define a field corresponding to the INFO field in a VCF file, Viola will 108 automatically generate INFO fields such as SV length and type. Additionally, new INFO fields can be added 109 using BED file annotation and microhomology prediction. BED files can be used to annotate genes, CFSs, 110 replication timing, copy numbers, etc., which individually or in combination can be used to classify SVs.

\section{3. Application}

\section{$113 \quad 3.1$ Matrix Generation with Simple Code}

114 We ran Viola to generate an SV feature matrix using public BEDPE files reported in a PCAWG study (Li et 115 al., 2020). First, we downloaded 2,748 BEDPE files from the ICGC data portal and used Viola to read 2,605 116 of these files that were not empty as a MultiBedpe instance. Second, the instance was successfully annotated 117 by CFSs and replication timing BED files that we built according to the PCAWG study. We defined 25 SV 118 classes according to CFSs, replication timing, and SV length and then generated a 2,605 $\times 25$ feature matrix. 119 These operations were written in only 11 lines of the Python code, excluding code for custom SV definitions 120 (Supplementary Figure S2A). The matrix generated here can be easily reproduced by following the tutorial in 121 the Viola official document.

\subsection{Signature Extraction Analysis}

124 We extracted nine SV signatures from the generated matrix using a function of Viola that simultaneously 125 performs non-negative matrix factorization (NMF) and cluster stability evaluation (Supplementary Figures S3 
126 and S4). Several signatures, including the signatures of CFSs, small deletions ( $<50 \mathrm{~kb})$, and small duplications

$127(<50 \mathrm{~kb})$, were comparable to those in the PCAWG study (Li et al., 2020). We further explored the

128 association between each of the nine signatures and driver mutations of three well-known DNA repair genes:

129 BRCA1, BRCA2, and CDK12 (Supplementary Table S1). These genes were significantly associated with the

130 small duplication signature, small deletion signature, and medium-large duplication signature, as expected

131 from previous studies (Li et al., 2020; Menghi et al., 2018; Nik-Zainal et al., 2016; Popova et al., 2016)

132 (Supplementary Table S1).

133

\section{$134 \quad 3.3$ Multicaller VCF Merging}

135 We synthesized VCF files that mimicked the output from Manta, Delly, Lumpy, and Gridss. These files 136 shared several SVs recorded with errors within $100 \mathrm{bp}$ of each other. Four VCF files were read as the object 137 of Viola and then merged, with 100 bp being specified as the option for proximity. The identifier was added 138 as a new INFO and the same SVs were given the same ID. We removed SV records called by only one SV 139 caller. Finally, all shared SVs were merged as expected and successfully exported as a VCF file 140 (Supplementary Data 1).

\section{$142 \quad 3.4$ Annotation Performance}

143 We tested the performance of the annotations on 2,605 BEDPE files using 18 lines of CFS BED files. In total, 144618,492 break-ends were annotated according to whether each was present or absent on the CFS. On average, 145 this took 7.5 min to complete using a single thread on an Ubuntu x86_64 server (Intel Core i7-8700K CPU at $146 \quad 3.70 \mathrm{GHz})$.

\section{4. Conclusion}

149 We developed Viola, a tool for SV signature analysis that allows highly customizable SV classification. This 150 tool also overcomes the difficulty of parsing current VCF files as well as the problem of different notations 151 derived from different callers. Viola will help stimulate research in the SV field to better understand the 152 biological significance of SVs. 
154 We thank Enago (www.enago.jp) for the English language review.

155 Financial Support: This work was supported by AMED P-CREATE (JP20cm0106551) and KAKENHI

156 Grant-in-Aid for Scientific Research (A) (16H02481) to S. Ishikawa.

157 Conflict of Interest: none declared.

\section{References}

159 Cameron,D.L. et al. (2019) Comprehensive evaluation and characterisation of short read general-purpose 160 structural variant calling software. Nat. Commun., 10, 3240.

161 Cameron,D.L. et al. (2017) GRIDSS: sensitive and specific genomic rearrangement detection using positional 162 de Bruijn graph assembly. Genome Res., 27, 2050-2060.

163 Beroukhim,R. et al. (2010) The landscape of somatic copy-number alteration across human cancers. Nature, $164 \quad 463,899-905$.

165 Chen,X. et al. (2016) Manta: rapid detection of structural variants and indels for germline and cancer 166 sequencing applications. Bioinformatics, 32, 1220-1222.

167 Kuzniar,A. et al. (2020) sv-callers: a highly portable parallel workflow for structural variant detection in 168 whole-genome sequence data. PeerJ, 8, e8214.

169 Layer,R.M., Chiang,C., Quinlan,A.R. and Hall,I.M. (2014) LUMPY: a probabilistic framework for structural 170 variant discovery. Genome Biol., 15, R84.

171 Li,Y. et al. (2020) Patterns of somatic structural variation in human cancer genomes. Nature, 578, 112-121.

172 Menghi,F. et al. (2018) The tandem duplicator phenotype is a prevalent genome-wide cancer configuration 173 driven by distinct gene mutations. Cancer Cell, 34, 197-210.e5.

174 Mills,R.E. et al. (2011) Mapping copy number variation by population-scale genome sequencing. Nature, 470, $17559-65$.

176 Nik-Zainal,S. et al. (2016) Landscape of somatic mutations in 560 breast cancer whole-genome sequences.

177 Nature, 534, 47-54.

178 Popova,T. et al (2016) Ovarian cancers harboring inactivating mutations in CDK12 display a distinct genomic 179 instability pattern characterized by large tandem duplications. Cancer Res., 76, 1882-1891. 
180 Rausch,T. et al (2012) DELLY: structural variant discovery by integrated paired-end and split-read analysis.

181 Bioinformatics, 28, i333-i339.

182 Thutkawkorapin,J. (2020) pyCancerSig: subclassifying human cancer with comprehensive single nucleotide,

183 structural and microsatellite mutational signature deconstruction from whole genome sequencing. BMC

184 Bioinformatics, 21, 128.

185 Wickham,H. (2014) Tidy Data. J. Stat. Softw., 59, 1-23.

186

\section{Supplementary Information}

Structure of VCF file

\begin{tabular}{|c|c|c|c|c|c|c|c|c|c|c|}
\hline \#CHROM & POS & ID & REF & ALT & QUAL & FILTER & INFO & FORMAT & SAMPLE01 & SAMPLE02 \\
\hline chr1 & 10 & id1 & A & $<D E L>$ & 60 & PASS & $E N D=20 ; S V L E N=10 ; S V T Y P E=D E L ; C I P O S=-1,1 ; C I E N D=-1,1$ & SU:PR:SR & $10: 5: 5$ & $6: 2: 4$ \\
\hline chr2 & 20 & id2_1 & C & C $[$ chr3:30[ & 10 & LowQual;MaxMQ0Frac & SVTYPE=BND;CIPOS=-1,2;MATEID=id2_2 & SU:PR:SR & 0:0:0 & $20: 4: 16$ \\
\hline chr3 & 30 & id2_2 & $T$ & ]chr2:20] T & 10 & LowQual;MaxMQ0Frac & SVTYPE=BND;CIPOS=-2,2;MATEID=id2_1 & SU:PR:SR & 0:0:0 & $23: 10: 13$ \\
\hline
\end{tabular}

Structure of viola.Vcf class

Positions Table

\begin{tabular}{|r|r|r|r|r|r|r|r|r|r|}
\hline id & chrom1 & pos1 & chrom2 & pos2 & strand1 & strand2 & ref & alt & svtype \\
\hline \hline id1 & chr1 & 10 & chr1 & 21 & + & - & A & $<$ DEL $>$ & DEL \\
\hline id2_1 & chr2 & 20 & chr3 & 30 & + & - & C & C[chr3:30[ & BND \\
\hline id2_2 & chr3 & 30 & chr2 & 20 & - & + & T & ]chr2:20]T & BND \\
\hline
\end{tabular}

Filters Table

\begin{tabular}{|r|r|}
\hline id & filter \\
\hline \hline id1 & PASS \\
\hline id2_1 & LowQual \\
\hline id2_1 & MaxMQ0Frac \\
\hline id2_2 & LowQual \\
\hline id2_2 & MaxMQ0Frac \\
\hline
\end{tabular}

Formats Table

\begin{tabular}{|r|r|r|r|r|}
\hline id & sample & format & value_idx & value \\
\hline \hline id1 & SAMPLE01 & SU & 0 & 10 \\
\hline id1 & SAMPLE01 & PR & 0 & 5 \\
\hline id1 & SAMPLE01 & SR & 0 & 5 \\
\hline id1 & SAMPLE02 & SU & 0 & 6 \\
\hline id1 & SAMPLE02 & PR & 0 & 2 \\
\hline id1 & SAMPLE02 & SR & 0 & 4 \\
\hline id2_1 & SAMPLE01 & SU & 0 & 0 \\
\hline id2_1 & SAMPLE01 & PR & 0 & 0 \\
\hline id2_1 & SAMPLE01 & SR & 0 & 0 \\
\hline id2_1 & SAMPLE02 & SU & 0 & 20 \\
\hline id2_1 & SAMPLE02 & PR & 0 & 4 \\
\hline id2_1 & SAMPLE02 & SR & 0 & 16 \\
\hline id2_2 & SAMPLE01 & SU & 0 & 0 \\
\hline id2_2 & SAMPLE01 & PR & 0 & 0 \\
\hline id2_2 & SAMPLE01 & SR & 0 & 0 \\
\hline id2_2 & SAMPLE02 & SU & 0 & 23 \\
\hline id2_2 & SAMPLE02 & PR & 0 & 10 \\
\hline id2_2 & SAMPLE02 & SR & 0 & 13 \\
\hline
\end{tabular}

\section{INFO fields Table}

\begin{tabular}{|r|r|r|}
\hline id & value_idx & end \\
\hline \hline id1 & 0 & 20 \\
\hline \multicolumn{3}{|c|}{} \\
\hline id & value_idx & svlen \\
\hline \hline id1 & 0 & 10 \\
\hline
\end{tabular}

\begin{tabular}{|r|r|r|}
\hline id & value_idx & svtype \\
\hline \hline id1 & 0 & DEL \\
\hline id2_1 & 0 & BND \\
\hline id2_2 & 0 & BND \\
\hline
\end{tabular}
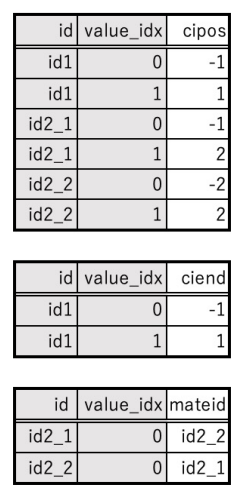

188 Supplementary Figure S1. Data structure of a viola.Vcf object. The upper part of the figure shows an

example of a Manta-like VCF. As shown in the lower part of the figure, the viola.Vcf object holds the 
190 information of a VCF file in several rectangular tables. The tables are related to each other by VCF IDs. The

191 grey columns are the primary key or composite primary key of the table. The header information of the VCF

192 is also stored as tables (not shown). Abbreviations: POS: start position of the SV; END: end position of the

193 SV; SVLEN: length of the SV; SVTYPE: type of SV; CIPOS: confidence interval around POS; CIEND:

194 confidence interval around END; MATEID: ID of mate break end; SU: count of supporting reads of the SV;

195 PR: count of paired end reads supporting the SV; SR: count of split reads supporting the SV.

\section{A signature_analysis_demo.ipynb}

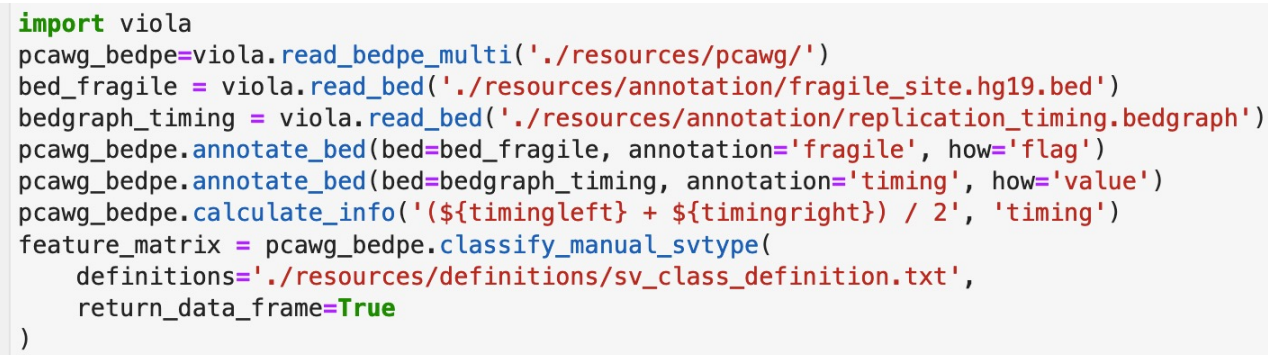

\section{CFile Tree}

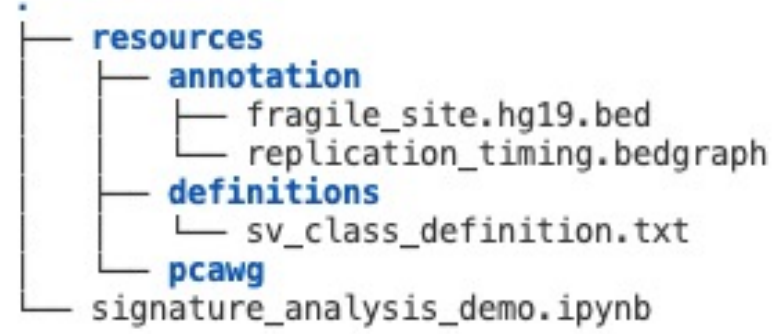

name 'At fragile site DEL'

0 fragileleft $==$ True

1 fragileright $==$ True

2 svtype $==$ DEL

logic $(0 \mid 1) \& 2$

name 'At fragile site DUP'

0 fragileleft $==$ True

1 fragileright $==$ True

2 svtype $==$ DUP

logic $(0 \mid 1) \& 2$

name ' $<50 \mathrm{~kb}$ early DEL'

0 svlen $>-50000$

\section{B sv_class_definition.txt}

(subset)

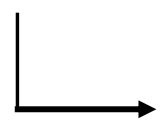

supe $=-$ DEL

logic $0 \& 1 \& 2$

name ' $<50 \mathrm{~kb}$ mid DEL'

0 svlen $>-50000$

1 timing $>33.35$

2 svtype $==$ DEL

logic $0 \& 1 \& 2$

name ' $<50 \mathrm{~kb}$ late DEL'

0 svlen $>-50000$

1 svtype $==$ DEL

logic 0 \& 1

Supplementary Figure S2. Example code for feature matrix generation. (A) (1) Import Viola package. (2)

Read BEDPE files under the "pcawg" directory as viola.MultiBedpe object. (3 and 4) Load common fragile

site and replication timing BED/BEDGRAPH for annotation*. (5 and 6) Annotate "pcawg_bedpe" variable

with the BED/BEDGRAPH loaded above. (7) Obtain mean replication timing for each SV breakpoint. (8-11)

Classify custom SV type according to the definition file and export feature matrix. (B) Definition file for

shown here is part of all SV definitions used in this study. (C) File tree of this analysis.

* Currently, a clear distinction between BED and BEDGRAPH files is not made in relation to the annotation 


\section{Signature Extraction Procedure}

208 Here, we describe how SV signatures were extracted from the PCAWG dataset. To determine the number

209 of signatures, $K$, we evaluated the stability of signatures derived from non-negative matrix factorization

210 (NMF) and its reconstruction error. Detailed steps are provided below.

212 1) Generate a new $2,605 \times 25$ matrix, $\dot{M}$, by bootstrapping the original matrix M. Here each element $\dot{m}_{i, j}$ 213 of $\dot{M}$ is chosen with a probability of $m_{i, j} / \sum_{i, j} m_{i, j}$, where $m_{i, j}$ is each element of $\mathrm{M}$ while $\sum_{i, j} \dot{m}_{i, j}=$ $214 \quad \sum_{i, j} m_{i, j}$

215 2) Apply NMF to the bootstrapped matrix $\dot{M}$ to obtain an exposure matrix, $\dot{E}$, with $2,605 \times K$ and a

219 3) Perform step 1 and 2 for 100 iterations to obtain $100 \mathrm{~K}$ signatures.

220 4) Use a K-means method for clustering $100 \mathrm{~K}$ signatures into $\mathrm{K}$ clusters with the constraint that signatures from the same iteration should not been assigned to the same cluster. The average silhouette score is calculated for stability evaluation.

5) The average signature matrix $\bar{P}$ is constructed with $K \times 25$. Each row of $\bar{P}$ is the centroid of the K-means clustering performed in step 4 . The average exposure matrix $\bar{E}$ is then calculated by NMF using the original matrix $\mathrm{M}$ and $\bar{P}$, where the matrix $\bar{P}$ is not updated while NMF. Finally, the Kullback-Leibler divergence of $\mathrm{M}$ and $\bar{E} \times \bar{P}$ was calculated as reconstruction error.

228 Steps 1-5 were conducted for each $K$ ranging from 2 to 13 (Supplementary Figure S3). 
Silhouette Score vs KL Divergence

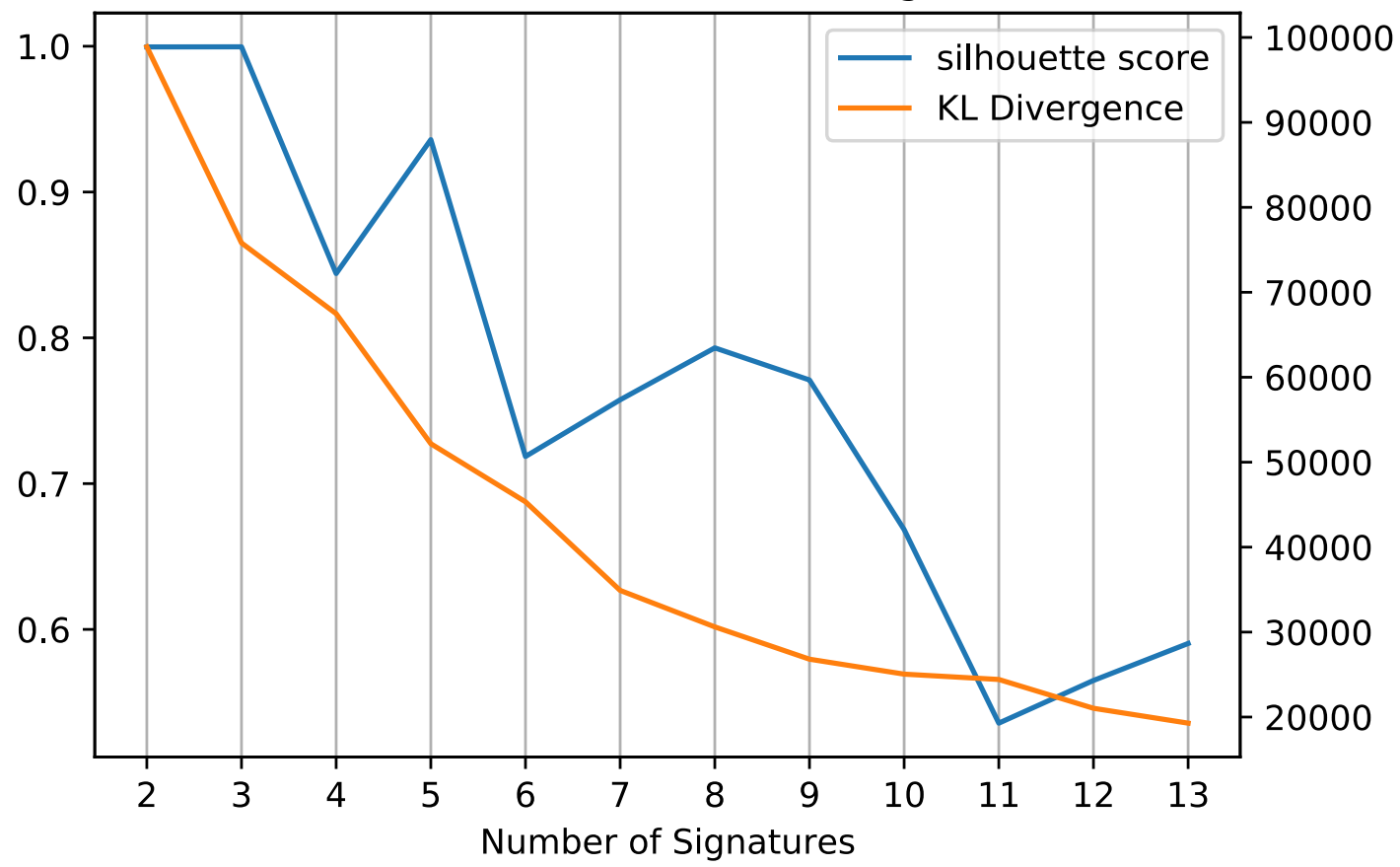

229 Supplementary Figure S3. Average silhouette score of K-means clusters and reconstruction error for

230 the number of signatures (K). After a manual assessment of each $\mathrm{K}$ with reference to the stability score and

231 reconstruction error, we chose $\mathrm{K}=9$ as the number of signatures. Extracted signatures are shown in 232 Supplementary Figure S4. 
Structural Variation Signatures

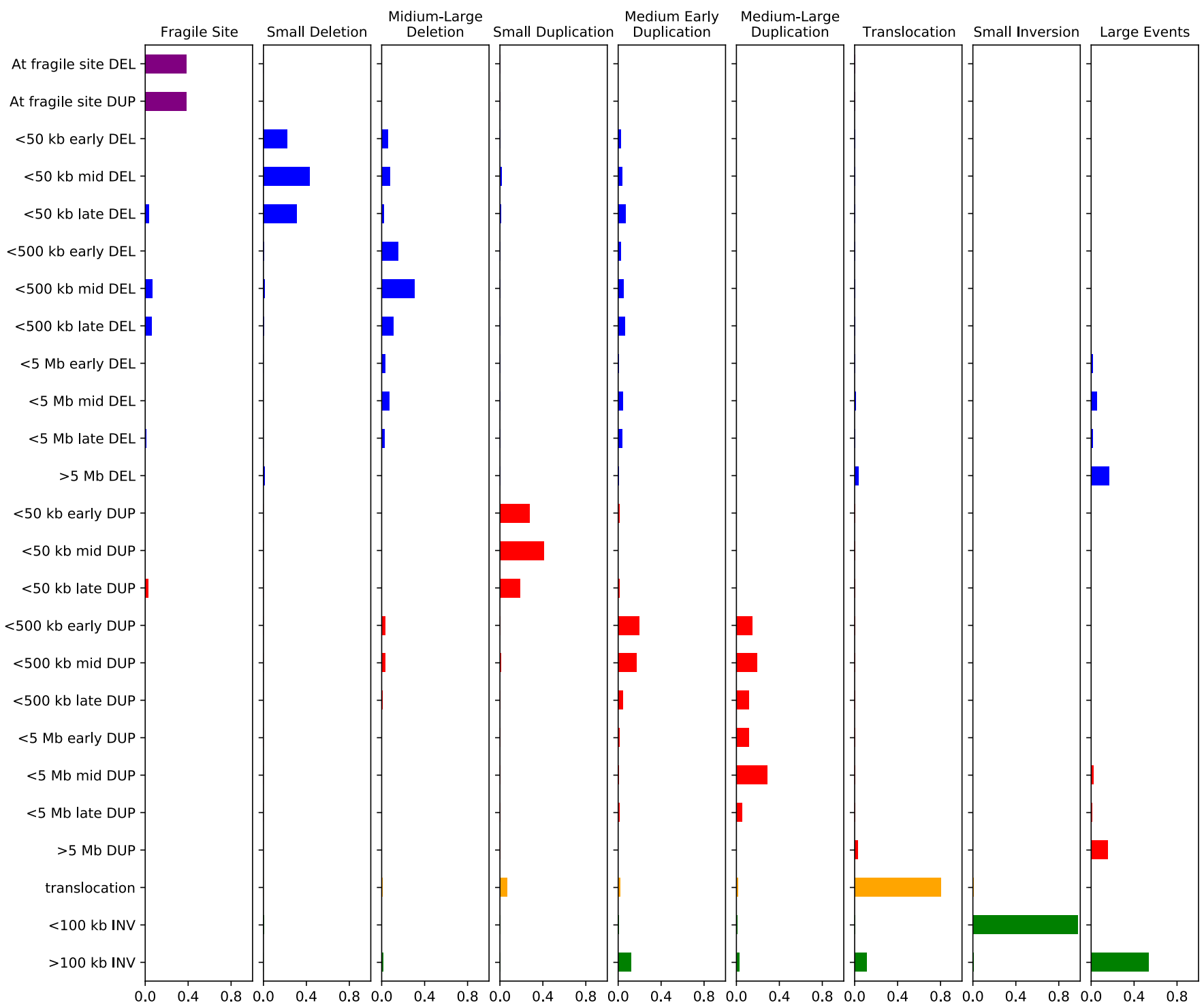

\section{Statistical testing of the association between signatures and driver mutations}

236 We obtained several signatures that were comparable with those in the PCAWG report such as the small

237 deletion signature and medium-large duplication signature. Statistical significance was tested for the effect of

238 driver mutations in BRCA1, BRCA2, and CDK12 on the nine signatures. The $\mathrm{p}$ value of each signature was

239 calculated using a linear model that considered the histological type of each sample (Supplementary Table 240 S1). 
242 Supplementary Table S1. Statistical significance of the effect of driver mutations in $B R C A 1, B R C A 2$,

243 and $C D K 12$ on nine signatures. Negative $\log p$ values are shown $\left({ }^{*} p<0.01\right)$.

\begin{tabular}{|c|c|c|c|}
\hline & BRCA1 & BRCA2 & CDK12 \\
\hline Fragile Site & 0.205 & 0.527 & 0.192 \\
\hline Small Deletion & 0.084 & $\mathbf{2 3 . 2 7 8}^{*}$ & 0.750 \\
\hline Medium-Large Deletion & 0.401 & 1.142 & 0.036 \\
\hline Small Duplication & $\mathbf{2 6 . 0 3 0}^{*}$ & 1.579 & 1.055 \\
\hline Medium Early Duplication & 0.389 & 0.421 & 0.057 \\
\hline Medium-Large Duplication & 0.663 & 1.526 & $\mathbf{6 . 2 5 1}^{*}$ \\
\hline Translocation & 0.122 & 0.218 & 0.950 \\
\hline Small Inversion & 0.729 & 0.520 & 0.128 \\
\hline Large Events & $\mathbf{2 . 8 7 7}^{*}$ & $\mathbf{2 . 0 4 2}^{*}$ & 0.479 \\
\hline
\end{tabular}

